\title{
Possible Ring Structures of Armchair Single-Walled Carbon Nanotubes
}

\author{
Venkatachalam Tamilmani§, Claude Daul ${ }^{*}$, and Titus Jenny \\ $\S S C S$ Poster Prize Winner
}

\begin{abstract}
Energetics and the electronic structure of various types of single-walled carbon nanotubes have been investigated by using Density Functional Theory. Armchair [n,n], zigzag [n,0] and chiral [n,m] $\mathrm{C}_{40} \mathrm{H}_{20}$ nanotubes have been considered. Calculations show that the armchair isomer is the most stable among the three types and they further reveal the factors that stabilize this isomer. Nucleus-independent chemical shift calculations indicate the aromaticity of the individual hexagonal rings in the carbon nanotubes and explain the extent of electron delocalization in them.
\end{abstract}

Keywords: Aromaticity $\cdot$ DFT $\cdot$ Nanotube $\cdot$ NBO $\cdot$ NICS

\section{Introduction}

Extensive research has been conducted both experimentally and theoretically on carbon nanotubes (CNT) since their discovery by the electron microscopy [1]. This discovery attracted physicists, chemists, nano technologists and material scientists in large numbers. More research has been devoted to their extraordinary electronic properties [2]. Many reports have appeared in the literature related to thermal and structural [3] studies that showed clear dependence of the nanotube properties on their diameter, length, and chirality [4]. Structures and aromaticity of finite-length armchair [5] CNT and the reactivity of carboxylic groups on armchair and zigzag CNT [6] have been reported. Clearly, nanotube research has become a booming area since their discovery [7]. In this paper, we address specifically the stability, the layout of the ends, and try to identify the factors that stabilize the isomers: armchair nanotubes $\left(\mathrm{n}=\mathrm{m}\right.$, chiral $\left.\angle 30^{\circ}\right)$, zigzag ( $\mathrm{n}$ or $\mathrm{m}=0$, chiral $\left.\angle 0^{\circ}\right)$ and chiral $(\mathrm{n} \neq \mathrm{m}$, chiral $\left.\angle 0-30^{\circ}\right)$. We have focused our interest on

\footnotetext{
${ }^{\star}$ Correspondence: Prof. Dr. C.A. Dau

University of Fribourg

Department of Chemistry

Chemin du Musée, 9

$\mathrm{CH}-1700$ Fribourg

Tel.: +4126300 8741

Fax: + 41263009738

E-Mail: claude.daul@unifr.ch
}

the energetics of the different geometries of armchair nanotube endings.

\section{Computational Details}

Density Functional Theory (DFT) calculations were performed using GAUSSIAN03 [8], Revision C.02 on a cluster of AMD Opteron (tm) Processors 246, 2.0 GB CPU. Geometry optimizations were carried out at B3LYP/6-31G(d). After performing a geometry optimization, Natural Bond Orbital (NBO) [9] and NICS [10] analysis have been carried out. NBO delocalization energetic analysis were performed using the \$DEL keyword as implemented in Gaussian. Total binding energy of a system can be partitioned into Lewis ( $\mathrm{E}_{\mathrm{L}}$ localized) and nonLewis $\left(\mathrm{E}_{\mathrm{NL}}\right.$ delocalized) contributions:

$$
\mathrm{E}_{\mathrm{NL}}=\mathrm{E}_{\mathrm{Tot}}-\mathrm{E}_{\mathrm{L}}
$$

The Rydberg and anti-bonding orbitals are the non-Lewis NBO orbitals that are denoted by a star (Ry*, BD*); these are deleted and NBO calculation performed. The difference in the energy of the system before and after deletion of anti-bonding terms provides a useful measure of the energy contribution due to the deleted terms. $\mathrm{E}_{\mathrm{NL}}$ (non-Lewis) energy is equivalent to $\mathrm{E}_{\text {deloc }}$ (delocalization energy). Using this approach, the delocalization energy was calculated.

NICS [10] is a simple and useful measurement to characterize the aromaticity of molecules. NICS was computed using the Gauge Including Atomic Orbitals (GIAO) method at the B3LYP/6-311+G(d,p) level that gives indices of the negative value of the magnetic shielding, computed at individual ring centre NICS(0), at $1 \AA$ distance above the individual ring centre NICS(1) and at the centre of the nanotube. NICS depends not only on the $\pi$ system but also on the contributions of lone pairs, atoms, and the circulation of electrons.

\section{Results and Discussion}

Possible ring structures of single-walled carbon nanotubes (SWCNT) are shown in Fig. 1a and $\mathrm{b}$.

The structures differ in their geometry; the difference is in the number of full benzenoid and repulsive hydrogen pairs. Therefore $\mathbf{F}, \mathbf{E}$ and $\mathbf{D}$ each contain five aromatic rings but different numbers of repulsive hydrogen pairs: 5, 4, and 3 respectively. Structures $\mathbf{C}$ and $\mathbf{B}$ have the same number of repulsive hydrogen pairs (two), but the number of full aromatic rings are four and three respectively. The $\mathbf{A}$ form has two full aromatic benzene rings and one repulsive hydrogen pair only. These results are listed in Table 1 along with their calculated relative energies, frontier orbital energy (FOE) gap, and delocalization energy. The corresponding bond lengths are given in Table 2. The curvature effect of armchair SWCNT is shown in Fig. 2.

Calculated energy values show that the armchair isomer is the most stable. Choosing this isomer as reference, relative energies of other isomers are presented in Table 1 . These values show that $\mathbf{A}$ is the least stable. Isomer $\mathbf{E}$ is less stable by $2.1 \mathrm{kcal} / \mathrm{mol}$ than $\mathbf{F}$. The 
a)

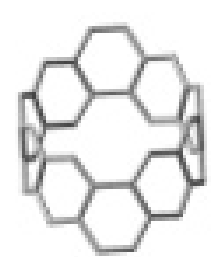

A

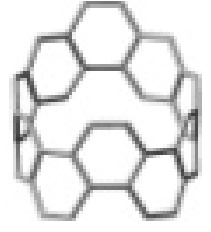

B

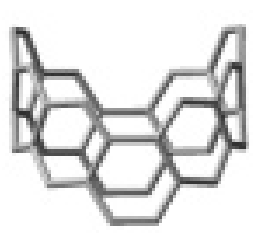

C

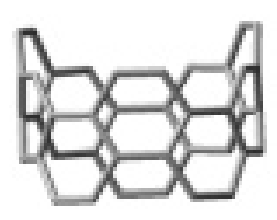

D

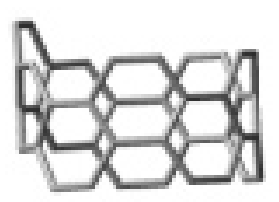

E

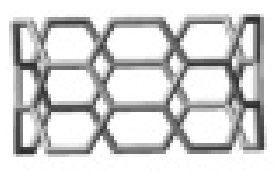

F

a)

b)

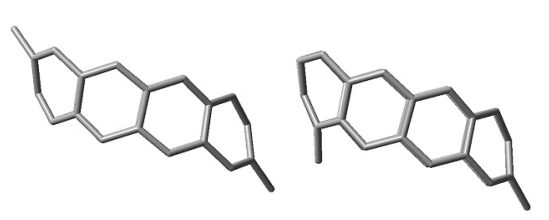

A

B

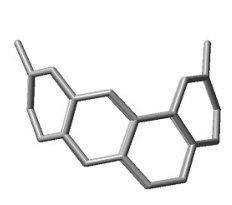

C

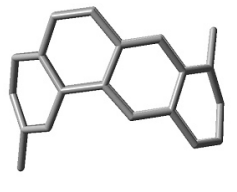

D

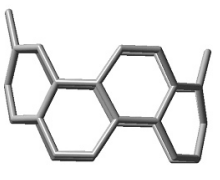

E

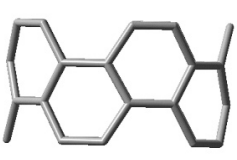

F

b)

c)



$\underset{\alpha-7.45}{\mathbf{A}}$

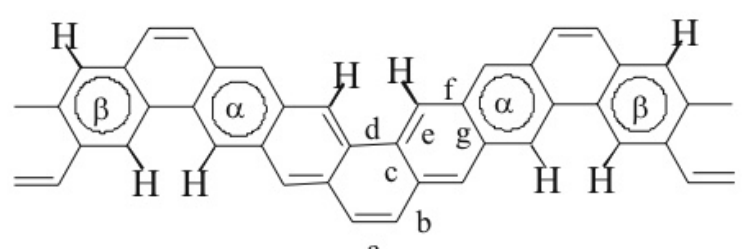

a

C

$\alpha-7.00$

$\beta-7.34$

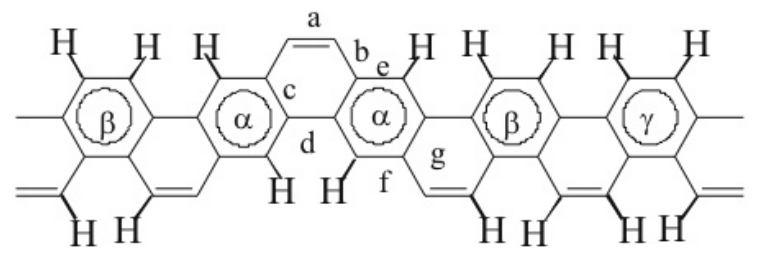

E

$\alpha-6.43$

$\beta-7.72$

$\gamma-5.71$

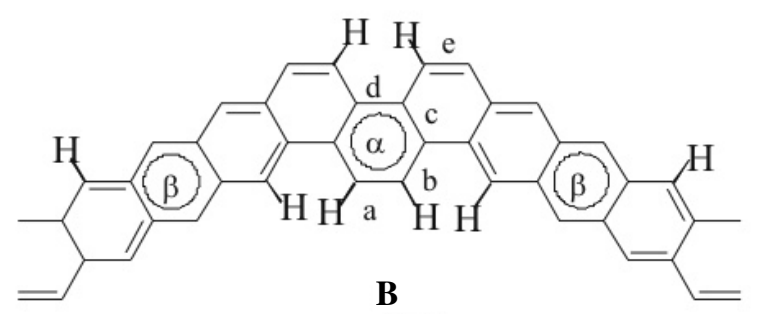

$\alpha-5.70$

$\beta-7.75$

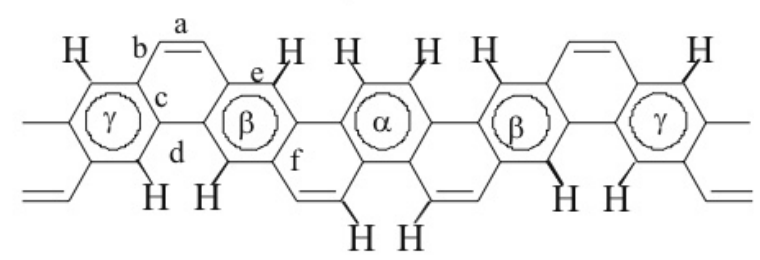

D

$\alpha-6.13$

$\beta-8.21$

$\gamma-7.32$

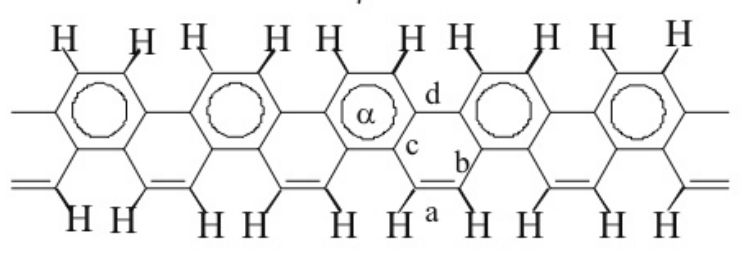

c) 
difference in energy is due to the number of $\mathrm{C}=\mathrm{C}$ outer bonds (marked in Fig. 1c as ' $\mathrm{a}$ ') in the molecule. Optimized structures show that in all the isomers the bond labeled as ' $a$ ' measures $1.35 \AA$; i.e. shorter than all other bonds. The bond labeled 'd' (Fig.1c) that connects the two rings is much longer $(1.452$ $\AA$ ) in isomer $\mathbf{F}$ and slightly longer for other isomers as shown in the Table 2. The Frontier Orbital Energy (FOE) gaps also reveal that the gap for the isomer $\mathbf{F}$ is larger than for the other isomers.

We also have evaluated the curvature effect of the $\mathbf{F}$ nanotube since it is the most stable one. We have taken a planar $\mathrm{C}_{42} \mathrm{H}_{24}$ molecule and calculations have been carried out at different radii and a good correlation with the mechanical strain model shown in Fig 2 was obtained. The same work has already been done using band structure calculations [11] and our DFT result is in good agreement with the literature value.

However it is still not clear what factors influence the stability of the geometries. Thus, we have extended our calculations to find out the delocalization energy of the molecule. NBO was used to evaluate the delocalization energy. According to the NBO procedure, calculations were carried out for all geometries and the results are in good agreement with their total energies. The computed delocalization energies are presented in Table 1. It shows that $\mathbf{F}$ is the most stable isomer with a delocalization energy of $2742.48 \mathrm{kcal} / \mathrm{mol}$. The second most stable geometry is $\mathbf{E}$ and the least stable one is $\mathbf{A}$, which is $9537.81 \mathrm{kcal} / \mathrm{mol}$ greater than isomer $\mathbf{F}$.

Positive NICSs denote anti-aromaticity and negative NICSs denote aromaticity. Depending on the ring environment the NICSs value change as shown in Fig. 1c. Calculations were performed at the individual benzenoid rings at NICS (1) and the values are indicated in Fig. 1c. Isomer $\mathbf{F}$ has five benzenoid rings; all rings fall in the same category $\alpha$ $(-5.69 \mathrm{ppm})$ and it shows that $\mathbf{F}$ has high aromaticity. Isomer $\mathbf{E}$ has three types of benzenoid rings which are denoted $\alpha(-6.43 \mathrm{ppm})$, $\beta(-7.72 \mathrm{ppm})$ and $\gamma(-5.71 \mathrm{ppm})$. Benzenoid ring $\beta$ is more aromatic compared to $\alpha$ and $\gamma$. Like isomer $\mathbf{E}, \mathbf{D}$ also contains three types of benzenoid rings in which $\beta$ rings have the high aromatic value of $-8.21 \mathrm{ppm}$. Isomers $\mathbf{C}$ and $\mathbf{B}$ have only two types of benzenoid rings, namely $\alpha$ and $\beta$ in which the $\beta$ rings have NICS values of $-7.34 \mathrm{ppm}$ and -7.75 ppm. Isomer $\mathbf{A}$ has two $\alpha$ benzenoid rings (-7.45 ppm). NICS calculations at centre of the geometry reveals a paratropic compound; the results are presented in Table 1. Isomer $\mathbf{F}$ is less (14.24 ppm) paratropic compared with other isomers: E (14.77 ppm), D (15.32 ppm), C (16.15 ppm), B (18.52 ppm) and A (27.27 ppm).

Table 1. Calculated relative energy ( $\mathrm{kcal} / \mathrm{mol})$, Frontier Orbital Energy gap (eV), delocalization energy $(\mathrm{kcal} / \mathrm{mol})$ and NICS(1) (ppm) at the center of the SWCNTs

$\begin{array}{lllllll}\begin{array}{l}\text { Iso- } \\ \text { mer }\end{array} & \begin{array}{l}\text { No. of full aro- } \\ \text { matic rings }\end{array} & \begin{array}{l}\text { No. of repul- } \\ \text { sive H pairs }\end{array} & \begin{array}{l}\text { Relative } \\ \text { energy }\end{array} & \text { FOE gap } & E_{\text {deloc }} & \begin{array}{l}\text { NICS at center } \\ \text { of the SWCNT }\end{array} \\ \text { A } & 2 & 1 & 28.58 & 1.82 & 12380.29 & -27.27 \\ \text { B } & 3 & 2 & 19.44 & 2.10 & 12019.96 & -18.52 \\ \text { C } & 4 & 2 & 9.82 & 2.56 & 10664.50 & -16.15 \\ \text { D } & 5 & 3 & 3.82 & 2.80 & 7578.06 & -15.31 \\ \text { E } & 5 & 4 & 2.1 & 2.91 & 2757.50 & -14.76 \\ \text { F } & 5 & 5 & 0.0 & 3.05 & 2742.48 & -14.24\end{array}$

Table 2: Selected C-C bond lengths $(\AA)$ in this SWCNTs

$\begin{array}{llllllll}\text { Isomer } & \mathrm{a} & \mathrm{b} & \mathrm{c} & \mathrm{d} & \mathrm{e} & \mathrm{f} & \mathrm{g} \\ \text { A } & 1.349 & 1.460 & 1.487 & 1.459 & 1.375 & 1.434 & 1.449 \\ \text { B } & 1.349 & 1.450 & 1.450 & 1.486 & 1.373 & - & - \\ \text { C } & 1.350 & 1.457 & 1.447 & 1.484 & 1.391 & 1.420 & 1.436 \\ \text { D } & 1.354 & 1.451 & 1.430 & 1.474 & 1.40 & 1.430 & - \\ \text { E } & 1.354 & 1.452 & 1.433 & 1.476 & 1.399 & 1.407 & 1.430 \\ \text { F } & 1.366 & 1.435 & 1.412 & 1.452 & - & - & -\end{array}$

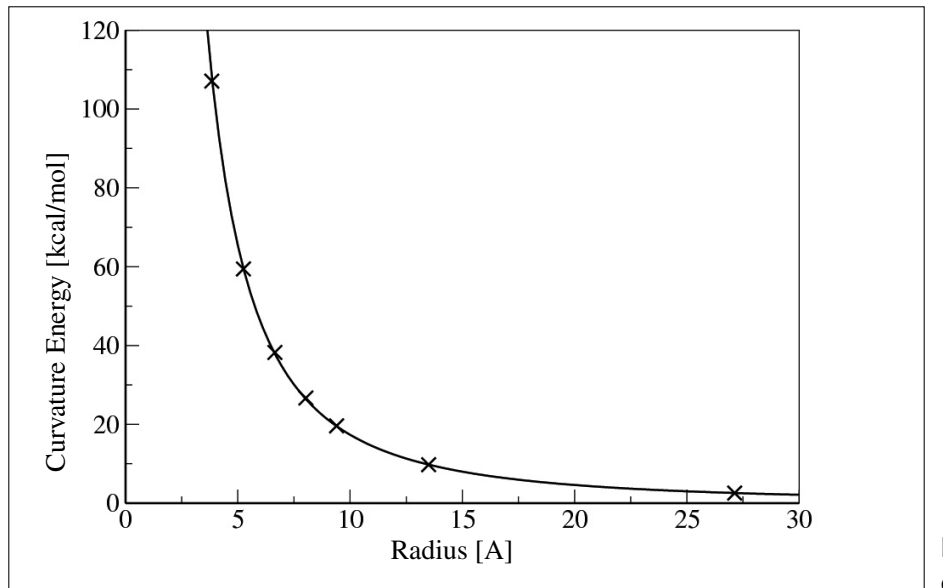

Fig. 2. Curvature effect on isomer $\mathbf{F}$ CNT

\section{Conclusions}

In summary, computed total energy, delocalization energy analysis, FOE gaps, NICS and the $\mathrm{C}-\mathrm{C}$ bond length clearly confirm that the $\mathbf{F}$ structure is the most stable. The other geometries are in the following decreasing order of stability $\mathbf{E}>\mathbf{D}>\mathbf{C}>\mathbf{B}>\mathbf{A}$.

\section{Acknowledgment}

This workhasbeen supported by Swiss National Science Foundation. V.T. thanks the Swiss Federal Commission for financial assistance as well as Clémence Corminbœuf for useful discussions.

\section{Received: January 27, 2006}

[1] S. Iijima, Nature 1991, 354, 56.

[2] a) R.A. Jishi, J. Bragin, L. Lou, Phys. Rev. $B$ 1999, 59, 9862; b) J. Cioslowski, N. Rao, D. Moncrieff, J. Am. Chem. Soc. 2002, 124 8485 ; c) A.S. Barnard, S.P. Russo, J. Phys. Chem. B 2003, 107, 7577 .

[3] S. Berber, Y.K. Kwon, D. Tomanek, Phys. Rev. Lett. 2000, 84, 4613 .

[4] a) J.W. Mintmire, B.I. Dunlap, C.T. White,
Phys. Rev. Lett. 1992, 68, 63; b) R. Saito, M. Fujita, M.S. Dresselhaus, G.R. Dresselhaus, Phys. Rev. B 1992, 46, 1804; c) N. Hamada, S. Sawada, A. Oshiyama, Phys. Rev. Lett. 1992, 68, 1579.

[5] Y. Matsuo, K. Tahara, E. Nakamura, Org. Lett. 2003, 18, 3181

[6] V.A. Basiuk, Nano Lett. 2002, 2, 835.

[7] M.S. Dresselhaus, G.R. Dresselhaus, R. Saito, Phys. Rev. B 1992, 45, 6234.

[8] Gaussian 03, Revision C.02, M.J. Frisch, Gaussian, Inc., Wallingford CT, 2004.

[9] a) T.K. Brunck, F. Weinhold, J. Am. Chem. Soc. 1978, 101, 1700; b) J.P. Foster, F. Weinhold, J. Am. Chem. Soc. 1980, 102, 7211; c) A.E. Reed, R.B. Weinstock, F. Weinhold, $J$. Chem. Phys. 1985, 83, 735; d) A.E. Reed, F. Weinhold, J. Chem. Phys. 1985, 83, 1736; e) A.E. Reed, L.A. Curtiss, F. Weinhold, $J$. Chem. Phys. 1985, 83, 1736.

[10] P.v.R. Schleyer, C. Maerker, A. Dransfeld, J.v.E. Jiao Hommes, J. Am. Chem. Soc. 1996, $118,6317$.

[11] O. Gülseren, T. Yildirim, S. Ciraci, Phys. Rev. B 2002, 65, 153405. 